\title{
Characterization of percolation behavior in conductor-dielectric 0-3 composites
}

\author{
Lin Zhang*, Patrick Bass*, Zhi-Min Dang ${ }^{\dagger}$ and Z.-Y. Cheng*,* \\ *Materials Research and Education Center \\ Auburn University \\ Auburn AL 36849, USA \\ ${ }^{\dagger}$ Laboratory of Dielectric Polymer Materials and Devices \\ School of Chemistry and Biological Engineering \\ University of Science and Technology Beijing \\ Beijing 100083, P. R. China \\ tchengzh@eng.auburn.edu
}

Received 30 September 2014; Accepted 17 November 2014; Published 5 December 2014

\begin{abstract}
The equation $\varepsilon_{\text {eff }} \propto\left(\phi_{c}-\phi\right)^{-s}$ which shows the relationship between effective dielectric constant $\left(\varepsilon_{\text {eff }}\right)$ and the filler concentration $(\varphi)$, is widely used to determine the percolation behavior and obtain parameters, such as percolation threshold $\varphi_{c}$ and the power constant $s$ in conductor-dielectric composites (CDCs). Six different systems of CDCs were used to check the expression by fitting experimental results. It is found that the equation can fit the experimental results at any frequency. However, it is found that the fitting constants do not reflect the real percolation behavior of the composites. It is found that the dielectric constant is strongly dependent on the frequency, which is mainly due to the fact that the frequency dependence of the dielectric constant for the composites close to $\varphi_{c}$ is almost independent of the matrix.
\end{abstract}

Keywords: Composite; dielectric constant; percolation; threshold; conductivity.

\section{Introduction}

From an application point of view, flexible dielectrics with a high dielectric constant are desirable. ${ }^{1-3}$ Among the dielectrics, polymers are flexible with a low dielectric constant (mostly $<5$ ), ${ }^{4}$ while inorganics can exhibit a high dielectric constant, ${ }^{5-7}$ but are brittle. Therefore, it is of interest to develop polymer-based composites, especially 0-3 composites due to the easiness of their preparation, in which a dielectric polymer is used as the matrix and filled with particles of other materials. ${ }^{8-10}$ A great deal of effort has been given to the development of composites with a relatively high dielectric constant in the last three decades. Based on the fillers used, the composites can be classified into two types: dielectric-dielectric composites (DDCs) in which the fillers are dielectric materials with a high dielectric constant; and conductor-dielectric composites (CDCs) where the fillers are conducting materials. ${ }^{8-11}$

For DDCs, the key is to find a filler material with a high dielectric constant. It is well known that polar dielectrics, such as ferroelectrics and relaxor ferroelectrics, exhibit a dielectric constant as high as $10^{3}$ to $10^{4} .^{7}$ Therefore, many composite systems have been developed and studied by using ferroelectric particles, such as $\mathrm{BaTiO}_{3}, \mathrm{PbTiO}_{3}, \mathrm{~Pb}(\mathrm{Zr}, \mathrm{Ti}) \mathrm{O}_{3}$, $\mathrm{Pb}\left(\mathrm{Mg}_{1 / 3} \mathrm{Nb}_{2 / 3}\right) \mathrm{O}_{3}{ }^{12-16}$ A dielectric constant close to 100 can be observed in those composites. Usually, in order to reach a dielectric constant of more than 50 , the composites need more than 50 vol.\% of the filler, which significantly reduces the flexibility of the composites. ${ }^{8}$ Additionally, the ferroelectrics have a dielectric constant that shows a strong temperature dependence, ${ }^{17}$ which is not desirable for many applications. Recently, some non-ferroelectric inorganic materials, which exhibit a high dielectric constant $\left(\sim 10^{4}\right)$ and weak temperature dependence, such as $\mathrm{CaCu}_{3} \mathrm{Ti}_{4} \mathrm{O}_{12}$, were discovered. ${ }^{18,19}$ When those dielectrics were used in the development of DDCs, a high dielectric constant of more than 500 can be observed. ${ }^{20}$ However, a high content $(>40$ vol. $\%$ ) of the filler is still needed. ${ }^{20-24}$

For the CDCs, their dielectric response is due to the percolation phenomena. That is, when the concentration $(\varphi)$ of filler is low, the composite is a dielectric. With increasing $\varphi$, the filler particles begin to form localized clusters and the dielectric constant increases. When the $\varphi$ approaches the percolation threshold $\left(\varphi_{c}\right)$, the dielectric constant of the composite increases abruptly and can reach a very high value. ${ }^{10,25}$ The CDCs can exhibit a much higher dielectric constant $(>1,000)$ at a lower content $(<10$ vol. \%) of filler particles when a lower $\varphi_{c}$ can be achieved. Therefore, a significant amount of research has been done in the development of CDCs. Various conductive materials, such as metal, carbon fibers, graphite, and carbon nanotubes have been used..$^{9-11}$ The $\varphi_{c}$ is the concentration at which the filler particles form infinitely conductive channels. That is, the $\varphi_{c}$

This is an Open Access article published by World Scientific Publishing Company. It is distributed under the terms of the Creative Commons Attribution 3.0 (CC-BY) License. Further distribution of this work is permitted, provided the original work is properly cited. 
for a composite system is an intrinsic parameter. The $\varphi_{c}$ is dependent on many factors, such as the size and shape of the filler particles, the distribution (i.e., connectivity) of filler particles, and the microstructure of the composite. ${ }^{9}$

The percolation behavior of the dielectric constant $\left(\varepsilon_{\text {eff }}\right)$ observed in the CDCs is widely described as ${ }^{9,11}$

$$
\varepsilon_{\text {eff }} \propto\left(\varphi_{c}-\varphi\right)^{-s},
$$

Or

$$
\varepsilon_{\text {eff }}=\varepsilon_{m}\left(\frac{\varphi_{c}-\varphi}{\varphi_{c}}\right)^{-s},
$$

where $\varepsilon_{m}$ is the dielectric constant of matrix, $s(>0)$ is a critical/power constant. Equation (1) was originally introduced for a random binary system with some approximations. $^{25}$ That is, this relationship is more suitable for 0-0 composites rather than 0-3 composites. Based on a numerical simulation of a random binary system, ${ }^{25}$ it was believed that $s \approx 0.7$ is a universal constant. From Eq. (1), one can find that the dielectric constant of composites can be used to determine/predict the values of $\varphi_{c}$ and $s$ (i.e., percolation behavior).

Equation (1) has been widely used in literature to determine the percolation behavior (i.e., the value of $\varphi_{c}$ and $s$ ) of 0-3 composites. $^{9-11}$ Based on the reported results, it is found that the value of $s$ determined using Eq. (1) actually changes over a broad range: as low as $0.167,{ }^{26}$ and as high as $1.78,{ }^{27}$ which is contradictory to the understanding that the $\mathrm{s}$ is a universal constant. Additionally, in a recent study based on $\mathrm{Ni}-\mathrm{P}(\mathrm{VDF}-\mathrm{TrFE})$ and Ni-P(VDF-CTFE) 0-3 nanocomposites, it is found that although Eq. (1) can be used to fit the relationship between the $\varphi$ and the $\varepsilon_{m}$ at a specific frequency, the fitting constants do not reflect the percolation behavior. $^{28,29}$ That is, the values of both $\varphi_{c}$ and $s$ are dependent on the selection of the dielectric constant used in the fitting, such as the frequency. In this paper, the dielectric constants from six CDC systems are used to check the suitability of Eq. (1) for 0-3 composites. The results confirm the conclusion that the fitting constants from Eq. (1) cannot be used to determine the percolation behavior of the 0-3 composites.

\section{Experimental Methods}

In this study, six CDC systems as shown in Table 1, which were reported previously, are studied. They are: System I - (PANI-PVDF), ${ }^{26}$ System II - (CB-BT-VMQ), ${ }^{30}$ System III - (Ag-PI), ${ }^{31}$ System IV - (CF-PVDF), ${ }^{32}$ System V - (TFP-MWNT-PVDF), ${ }^{33}$ and System VI (MWNT-PVDF). ${ }^{34}$ The first three systems use a spherical filler, while the last three use one-dimensional filler particles. Clearly, these composites have a low $\varphi_{\mathrm{c}}$ : from 1.6 to 12.2 vol.\%. From Table 1, one can find that the value of $s$ obtained using Eq. (1) for these composites changes over a big range (i.e., can be as low as 0.17 and as high as 1.6).
System I is a polyaniline/poly(vinylidene fluoride) (PANI/PVDF) composite prepared via a solution-grinding process and then hot-molding route. ${ }^{26}$ The average diameter and conductivity of PANI particles was $0.1 \mu \mathrm{m}$ and $6.7 \mathrm{~S} / \mathrm{m}$, respectively. The dielectric properties (i.e., dielectric constant and loss) were characterized at room temperature at frequencies from $100 \mathrm{~Hz}$ to $10 \mathrm{MHz}$. Using Eq. (1) with the dielectric constant at $100 \mathrm{~Hz}$, it was obtained that $\varphi_{\mathrm{c}}=0.045$ and $s=0.167$.

System II is a three-component high-elasticity nanocomposite, using methyl vinyl silicone rubber (VMQ) as a matrix and nanosized BT and carbon black (CB) as fillers. ${ }^{30}$ A silane coupling agent (KH550) was used to modify the surface of both BT and CB particles. Then the CB and BT were mixed with VMQ in tetrahydrofuran solvent and hot pressed to a disk shape. The size of $\mathrm{CB}$ was about $50 \mathrm{~nm}$ and surface area was about $60-70 \mathrm{~mm}^{2} / \mathrm{g}^{-1}$. The dielectric properties were characterized at room temperature at frequencies from $100 \mathrm{~Hz}$ to $10 \mathrm{MHz}$. It is found that the dielectric constant had a giant enhancement in the composites near the percolation threshold of around 0.035. Using Eq. (1) with the dielectric constant at $1 \mathrm{kHz}$, it was obtained that $\varphi_{c}=0.0375$ and $s=0.289$.

System III is a Ag/polyimide (PI) composite synthesized by using a simple ultrasonic dispersion and subsequent in situ polymerization processing technique. ${ }^{31}$ The $\mathrm{Ag}$ particles (0.5 $\mu \mathrm{m}$ in diameter), 4,4-diamino-diphenyl ether (ODA), and $\mathrm{N}$, N-dimethylacetamide (DMAc) were placed in a flask. After $0.5 \mathrm{~h}$ of ultrasonic dispersion, pyromellitic dianhydride and more DMAc were subsequently added. Finally, the yellow-colored, transparent Ag/PI hybrid films with a $30 \mu \mathrm{m}$ thickness were obtained after the mixture was magnetically stirred for $3 \mathrm{~h}$ at room temperature. The dielectric properties were characterized at room temperature over a frequency range from $1 \mathrm{kHz}$ to $10 \mathrm{MHz}$. Using Eq. (1) with the dielectric constant at $1 \mathrm{kHz}$, it is obtained that $\varphi_{c}=0.122$ and $s=0.27$.

System IV is an untreated conductive short carbon fiber (CF)/PVDF composite with low concentrations of CF added via a simple blending and hot-molding technique. ${ }^{32}$ The $\mathrm{CF}$ was the conducting filler with an average length and diameter of 100 and $8 \mu \mathrm{m}$, respectively. The dielectric properties were characterized at room temperature at frequencies from $1 \mathrm{kHz}$ to $1 \mathrm{MHz}$. Using Eq. (1) with the dielectric constant at $1 \mathrm{kHz}$, it was obtained that $\varphi_{c}=0.066$ and $s=0.87$.

System V is a PVDF-based nanocomposite with trifluorophenyl (TFP)-functionalized MWNTs used as fillers. ${ }^{33}$ The strong interaction between the PVDF and TFP-functionalized MWNTs was obtained by modifying it with 3,4,5-trifluorobromobenzene (TFBB). The film was folded and hot pressed at $200^{\circ} \mathrm{C}$ into a disk-shaped sample and further strengthened in the preferred orientation of the TFPMWNT in the matrix. The dielectric properties were characterized at room temperature at frequencies from $100 \mathrm{~Hz}$ to 
Table 1. Fitting parameters for five systems studied.

\begin{tabular}{|c|c|c|c|c|c|c|c|c|}
\hline \multirow[b]{2}{*}{ System } & \multirow[b]{2}{*}{ Composites } & \multirow[b]{2}{*}{ Shape } & \multirow[b]{2}{*}{ Size } & \multicolumn{3}{|c|}{ Literature } & \multicolumn{2}{|c|}{ This work } \\
\hline & & & & $\varphi_{c}(\%)$ & $s$ & Ref & $\varphi_{c}(\%)$ & $s$ \\
\hline I & PANI-PVDF & sphere & $0.1 \mu \mathrm{m}$ & 4.5 & 0.167 & 24 & $4.51-7.29$ & $0.149-5.332$ \\
\hline II & CB-BT-VMQ & sphere & $50 \mathrm{~nm}$ & 3.75 & 0.289 & 25 & $3.54-3.79$ & $0.275-0.304$ \\
\hline III & Ag-PI & sphere & $0.5 \mu \mathrm{m}$ & 12.2 & 0.27 & 26 & $12.15-12.24$ & $0.260-0.276$ \\
\hline IV & CF-PVDF & fiber & $\begin{array}{c}\mathrm{W}: 8 \mu \mathrm{m} \\
\mathrm{L}: 100 \mu \mathrm{m}\end{array}$ & 6.6 & 0.87 & 27 & $9.58-24.5$ & $0.697-2.626$ \\
\hline V & MWNT-PVDF & nanotube & $\begin{array}{l}\text { W: } 10-30 \mathrm{~nm} \\
\mathrm{~L}: 5-15 \mu \mathrm{m}\end{array}$ & 8 & 1.63 & 28 & $7.94-13.7$ & $0.765-5.738$ \\
\hline VI & MWNT-PVDF & nanotube & - & 1.61 & 0.31 & 29 & $1.61-1.83$ & $0.213-0.310$ \\
\hline
\end{tabular}

$10 \mathrm{MHz}$. Using Eq. (1) with the dielectric constant at $1 \mathrm{kHz}$, it was obtained that $\varphi_{c}=0.08$ and $s=1.63$.

System VI is an untreated multiwall carbon nanotubes/ poly(vinylidenefluoride) (MWNT/PVDF) composite synthesized by a simple physical blending with a hot-molding process. $^{34}$ The MWNT were ultrasonically dispersed in $\mathrm{N}, \mathrm{N}$-dimethylformamide (DMF) for as long as $2 \mathrm{~h}$ and the PVDF was dissolved in the DMF solvent at $50^{\circ} \mathrm{C}$. These two solutions were mixed and stirred by further ultrasonic treatment for $10 \mathrm{~min}$. Then, the solution was heated to $60^{\circ} \mathrm{C}$ for $8 \mathrm{~h}$ and consequently molded by hot-pressing at about $200^{\circ} \mathrm{C}$ and $15 \mathrm{MPa}$. The final samples were disk-shaped with a $12 \mathrm{~mm}$ diameter and a $1 \mathrm{~mm}$ thickness. A very low $\varphi_{c}(\sim 1.6$ vol.\% $)$ was observed in the composites. It was believed that the low percolation threshold is due to the large aspect ratio and the high conductivity of the MWNT. The dielectric properties were characterized at room temperature at frequencies from $100 \mathrm{~Hz}$ to $40 \mathrm{MHz}$. Using Eq. (1) with the dielectric constant at $1 \mathrm{kHz}$, it was obtained $\varphi_{c}=0.0161$ and $s=0.31$.

\section{Results and Discussion}

First of all, it was found that Eq. (1) can be used to fit the experimentally obtained relationship between the $\varphi$ and the $\varepsilon_{\text {eff }}$ at any frequency for all the composites studied here, as shown in Fig. 1, where the dielectric constant at two frequencies (i.e., $100 \mathrm{~Hz}$ and 105,953 Hz of System I was used). The same data and same equation were used to check the fitting results at different frequencies. For the fitting using the dielectric constant at $100 \mathrm{~Hz}$, it is obtained that $\varphi_{\mathrm{c}}=0.04504$ and $s=0.1705$, which are the same as the values reported in the original article. ${ }^{26}$ However, when the dielectric constant at $105,952 \mathrm{~Hz}$ was used in the fitting, it was obtained that $\varphi_{\mathrm{c}}=$ 0.05436 and $s=0.2174 \mathrm{~Hz}$. That is, comparing with the results obtained using the dielectric constant at $100 \mathrm{~Hz}$, the $\varphi_{\mathrm{c}}$ increases about $20 \%$ and the $s$ increases about $28 \%$. The increase in the $\varphi_{\mathrm{c}}$ and $s$ is much higher than the uncertainty ( $\sim 5 \%)$ of the fitting constants. That is, although Eq. (1) can be used to fit the experimental results, the fitting constants change with the selection of frequencies used. To further check this, the dielectric constant at different frequencies was used to perform the fitting. The results are given below.

System I: The fitting constants (i.e., $\varphi_{c}$ and $s$ ) obtained at different frequencies are plotted in Fig. 2(a). At frequencies below $10 \mathrm{kHz}$, as the frequency increases, the fitting constant $s$ slightly decreases, while the fitting constant $\varphi_{c}$ increases slightly. However, at high frequency range, both the $\varphi_{c}$ and $s$ rapidly increase with increasing frequency. When the frequency is close to $1 \mathrm{MHz}$, the values of $\varphi_{c}$ and $s$ can be more than four times of the values obtained at $100 \mathrm{~Hz}$.

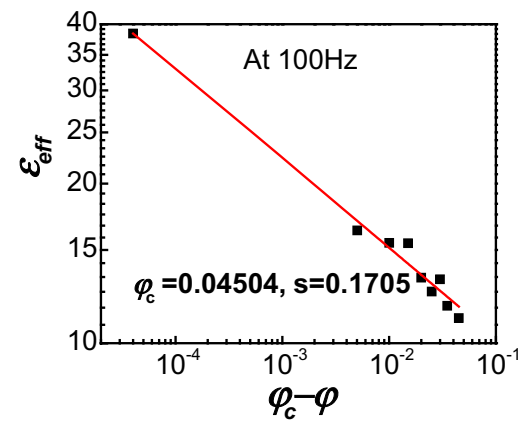

(a)

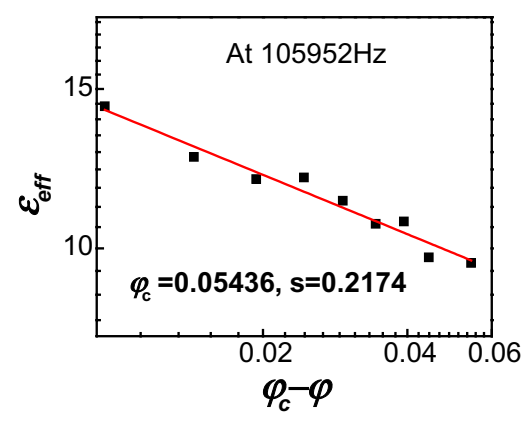

(b)

Fig. 1. $\varepsilon_{\text {eff }}$ versus volume fraction $\varphi$ and the fitting curve by Eq. (1) at different frequencies: (a) at $100 \mathrm{~Hz}$ and (b) $105,952 \mathrm{~Hz}$ for System I. 


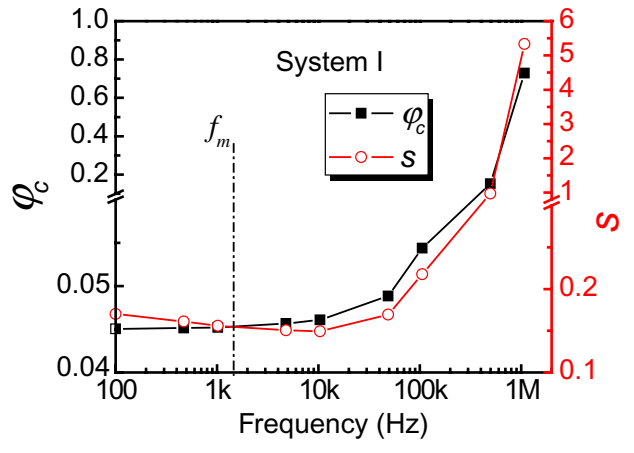

(a)

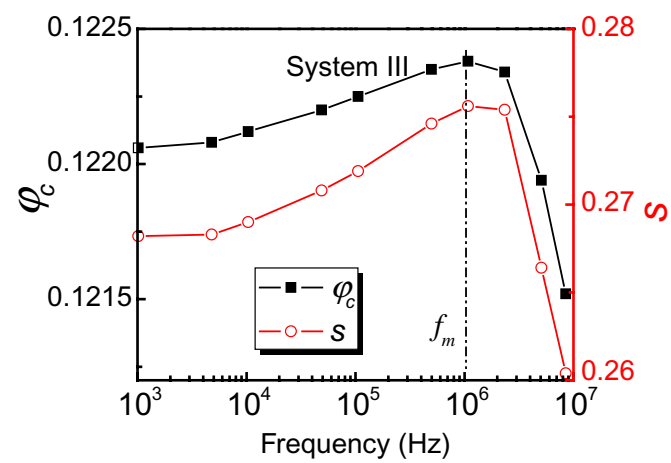

(c)

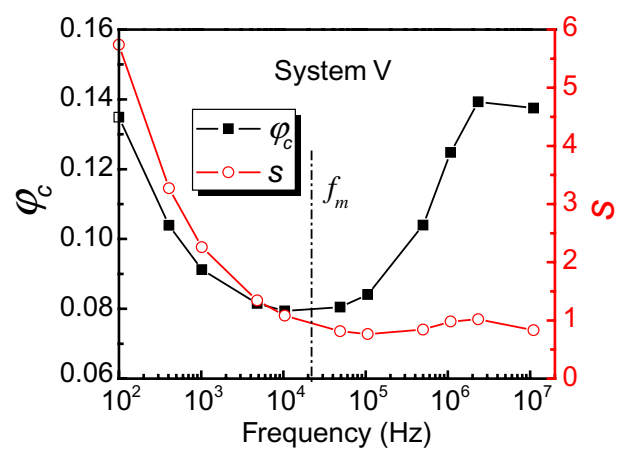

(e)

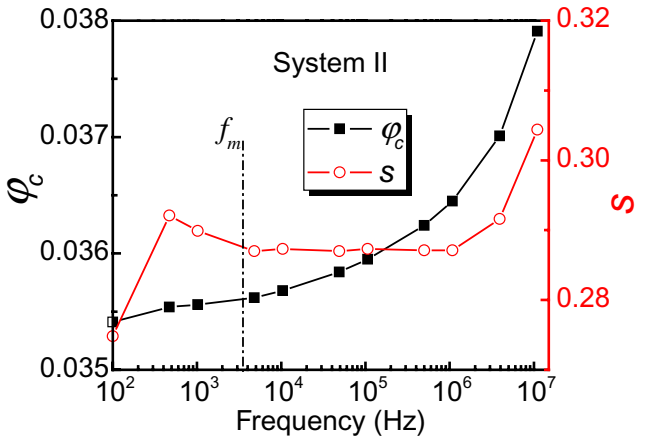

(b)

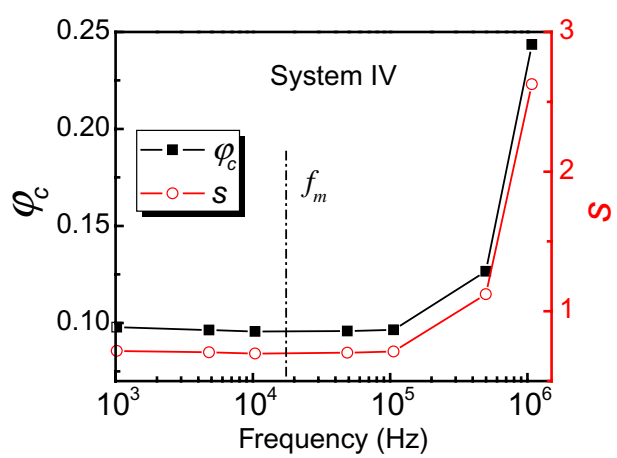

(d)

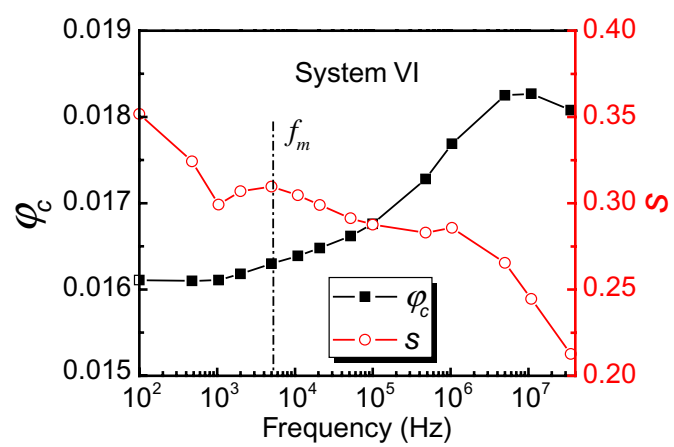

(f)

Fig. 2. Dependence of percolation threshold $\left(\varphi_{c}\right)$ and critical constant $(s)$ on frequency of six different systems: (a) System I, (b) System II, (c) System III, (d) System IV, (e) System V, and (f) System VI.

System II: The fitting constants obtained at different frequencies are shown in Fig. 2(b). Clearly, the $\varphi_{\mathrm{c}}$ continuously increases with increasing frequency. It is found that the $s$ obtained at $100 \mathrm{~Hz}$ is significantly lower than that obtained at other frequencies.

System III: The trend of System III is much more different than with Systems I and II. As shown in Fig. 2(c), both fitting constants $\varphi_{c}$ and $s$ slightly increase with increasing frequency until $1 \mathrm{MHz}$. At frequencies higher than $1 \mathrm{MHz}$, both fitting constants rapidly decease with increasing frequency.

It should be mentioned that the shape of the fillers used in Systems I, II, and III was the same (i.e., spherical), but the filler particles had different sizes: System I (diameter of
PANI $\sim 0.1 \mu \mathrm{m}$ ), System II (diameter of CB $\sim 50 \mathrm{~nm}$ ), and System III (diameter of $\mathrm{Ag} \sim 0.5 \mu \mathrm{m}$ ). However, the size of these particles is much smaller than the size of the sample used in the experiments. Therefore, not much influence on the percolation behavior is expected for the size of the filler particle. Based on the filler materials a big difference in the electric conductivity is expected for these three types of fillers. It is known that the relaxation process is induced in a composite due to the conductivity difference between two constitutes. Therefore, the difference in the electric conductivity of the filler may be related to the different dependences of the fitting constant on the frequency as shown in Fig. 2. 
System IV: As shown in Fig. 2(d), the fitting constants $\varphi_{c}$ and $s$ slightly decrease (almost a constant) with increasing frequency at low frequencies. At $1 \mathrm{kHz}$, it is found that $\varphi_{c}=$ 0.074 and $s=0.39$, which are different with the original report $\left(\varphi_{c} \approx 0.066\right.$ and $\left.s \approx 0.87\right) .{ }^{32}$ When the frequency is higher than $100 \mathrm{kHz}$, both fitting constants rapidly increase with increasing frequency, the fitting constant $\varphi_{c}$ and $s$ at $1 \mathrm{MHz}$ is about three times of that at $1 \mathrm{kHz}$.

System V: As shown in Fig. 2(e), the fitting constant $s$ continuously decreases with increasing frequency. There is a rapid decrease in the $s$ at low frequency. $s$ at $100 \mathrm{~Hz}$ is about six times compared to at $10 \mathrm{MHz}$. However, the fitting constant $\varphi_{c}$ shows a very different frequency dependence. At frequencies lower than $10 \mathrm{kHz}$, the $\varphi_{c}$ decreases with increasing frequency. At frequencies higher than $10 \mathrm{kHz}$, the $\varphi_{c}$ shows a rapid increase with increasing frequency. It should be mentioned that the fitting constant at $1 \mathrm{kHz}$ are $\varphi_{c}=0.091$ and $s=2.26$, which are different with the original report $\left(\varphi_{c}=0.08\right.$ and $\left.s=1.63\right){ }^{33}$

System VI: The fitting constants at $1 \mathrm{kHz}$ are $\varphi_{c}=$ 0.0161 and $s=0.299$, which are very close to the original report $\left(\varphi_{c}=0.0161\right.$ and $\left.s=0.31\right) .{ }^{34}$ As shown in Fig. 2(f), the fitting constant $\varphi_{c}$ increases with increasing frequency until $10 \mathrm{MHz}$. The fitting constant $s$ decreases with increasing frequency.

Particles with a 1D shape were used as the filler in Systems IV, V, and VI. Among these three systems, System VI exhibits the lowest $\varphi_{c}$, which may be related to the fact that the filler particles had the highest aspect ratio $(10-30 \mathrm{~nm}$ in diameter and $5-15 \mu \mathrm{m}$ in length). Besides the difference in the fitting constant $\varphi_{c}$, it is clearly observed that the frequency dependence of the fitting constants $\varphi_{c}$ and $s$ is also different among these three systems. These three systems exhibit very different frequency dependencies on the fitting constants $\varphi_{c}$ and $s$.

Based on the results shown in Fig. 2, one can conclude that the fitting constants $\varphi_{c}$ and $s$ are dependent on the frequency and there is no general trend on the frequency dependence of these two constants. Therefore, the fitting constant $\varphi_{c}$ cannot be used to determine/define the percolation threshold of the composites since as an intrinsic parameter of the composites the percolation threshold should be independent of the selection of data (i.e., dielectric constant) used in the analysis. From data shown in Fig. 2, one can find that the value of fitting constant $s$ can be as small as 0.15 and as high as 5 , which is significantly different with the current understanding that the $s$ is a universal constant of $\sim 0.7$. In some systems (such as Systems I and IV), one may expect a saturated value for the fitting constant at low frequency. However, this kind of saturated value is not expected for some systems (such as System V) based on the data shown in Fig. 2. Therefore, the data shown in Fig. 2 clearly demonstrates that the fitting constants $\varphi_{c}$ and $s$ from Eq. (1) do not reflect the percolation behavior of the CDCs.

The frequency dependence of the fitting constants $\varphi_{c}$ and $s$ originates from the frequency dependence of the dielectric

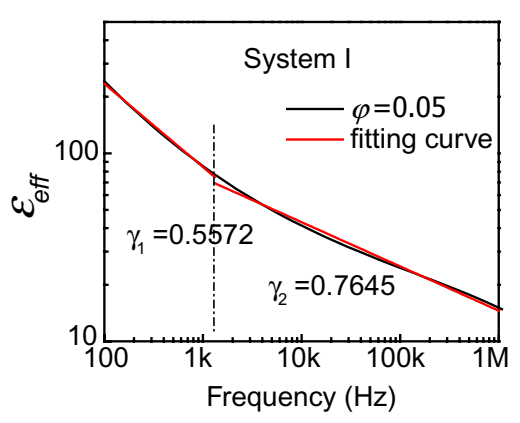

(a)

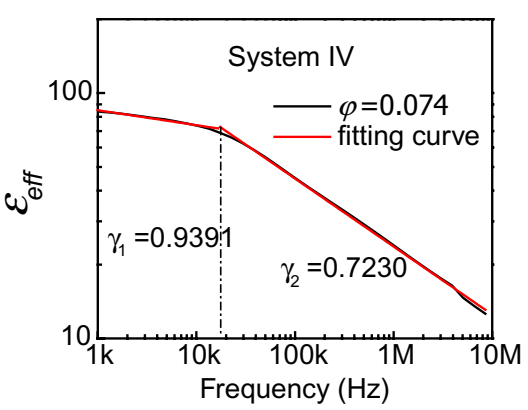

(d)

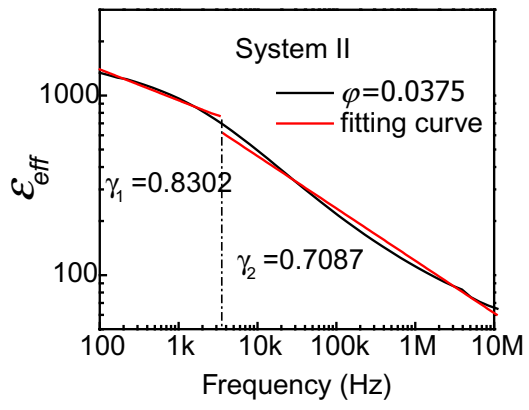

(b)

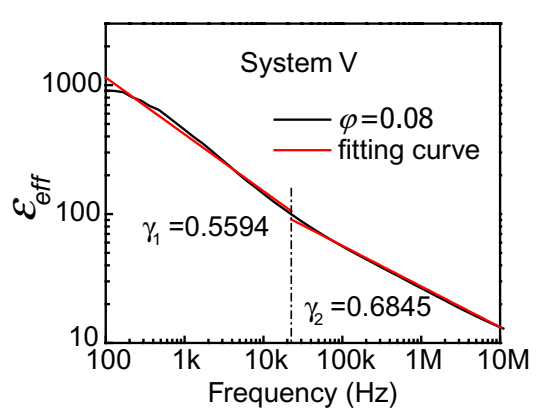

(e)

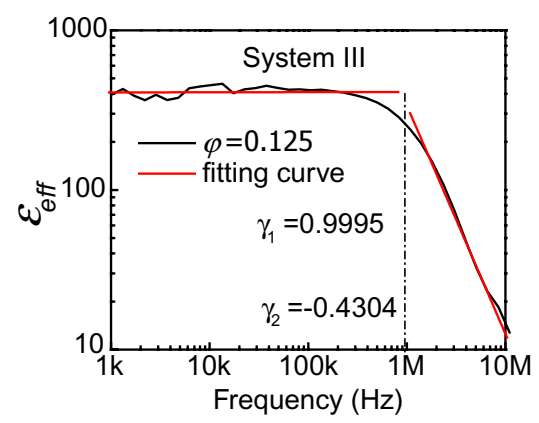

(c)

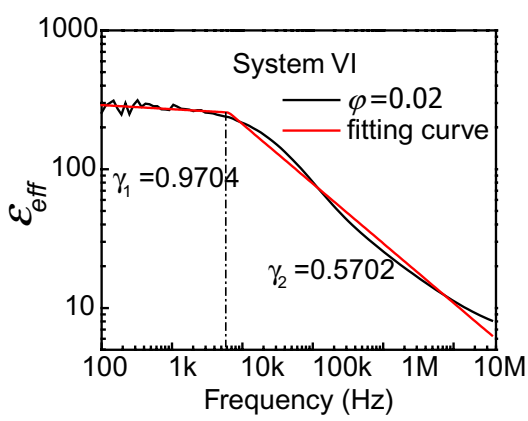

(f)

Fig. 3. Dielectric constant versus frequency of composites and fitting curve (fitted by Eq. (2)) of six different systems: (a) System I, (b) System II, (c) System III, (d) System IV, (e) System V, and (f) System VI. 
constant of the composites. There are a few possible processes that are responsible for the frequency dependence of the dielectric constant: (1) the $\varepsilon_{m}$, (2) new relaxation process induced by the mixing of different materials (i.e., Maxwell-Wagner effect), (3) percolation feature. In Eq. (1), the frequency dependence of $\varepsilon_{m}$ has been included. For the Maxwell-Wagner effect, it is known that the corresponding relaxation time is very small. ${ }^{28}$ Therefore, the MaxwellWagner effect would not affect the dielectric constant at the frequencies studied here. For the percolation process, it is known that the CDCs with a composition close to the $\varphi_{c}$ the dielectric constant can be described by ${ }^{35}$ :

$$
\varepsilon_{\text {eff }} \propto \omega^{\gamma-1},
$$

where $\gamma(<1)$ is a constant and $\omega$ is the angular frequency. For a random resistance network, it was indicated that $\gamma \approx 0.73 \pm 0.05,{ }^{35}$ while for a random binary medium, it was indicated that $\gamma=0.75 \pm 0.05 .^{36}$ It is known that, the value of " $\varepsilon_{\text {eff }} / \varepsilon_{m}$ " for CDCs with a composition much lower than $\varphi_{c}\left(\varphi \ll \varphi_{c}\right)$ is almost frequency independent. However, based on Eq. (2), the CDCs exhibit a strong dependence on the dielectric constant on the frequency when $\varphi$ is close to $\varphi_{c}\left(\varphi \sim \varphi_{c}\right)$. Therefore, it is expected that the fitting constant $\varphi_{c}$ using Eq. (1) should increase with increasing frequency. This is true for most of the cases as shown in Fig. 2.

When Eq. (2) is used to analyze the dielectric constant of these six CDC systems for the composites with $\varphi \sim \varphi_{c}$, it is found that Eq. (2) can be used to fit the experimental results, but there are two frequency regimes as shown in Fig. 3. The fitting constant $\gamma$ at low frequency regime is higher than that at high frequency regime. Interestingly, it is found that the fitting constant $\gamma$ at high frequency regime is around $0.7-0.8$ for these systems (except Systems III and VI), which is very close to the universal value of $\gamma$ (i.e., $\gamma=0.75$ ).

The frequency $\left(f_{m}\right)$ that separates two frequency regimes is different: $\sim 1 \mathrm{kHz}$ for System I, $3 \mathrm{kHz}$ for System II, $\sim 1 \mathrm{MHz}$ for System III, $\sim 20 \mathrm{kHz}$ for System IV, $\sim 20 \mathrm{kHz}$ for System V, and $\sim 7 \mathrm{kHz}$ for System VI. If this frequency (i.e., $f_{m}$ ) is used to compare with the results shown in Fig. 2, although there are some differences among the frequency dependence of the fitting constant $\varphi_{c}$ and $s$ for these six systems, it seems that the $f_{m}$ from Fig. 3 can be used to classify the frequency dependence of the fitting constants $\varphi_{c}$ and $s$ shown in Fig. 2. That is, the frequency dependence of the fitting constants $\varphi_{c}$ and $s$ from Eq. (1) is mainly due to the percolation behavior described by Eq. (2).

\section{Conclusions}

In conclusion, the widely used percolation equation, Eq. (1), was used to fit the dielectric constant of six CDC systems. It is found that although the equation can be used to fit the experimental data, the fitting constants $\varphi_{c}$ and $s$ do not reflect the percolation behavior of the composite system. That is, the fitting constants are dependent on the frequency selected. It is concluded that the problem originates from the percolation feature described by Eq. (2).

\section{Acknowledgment}

This work was supported by an USDA grant and an AU-IGP grant.

\section{References}

${ }^{1}$ R. E. Newnham, Composite electroceramics, Annu. Rev. Mater. Sci. 16, 47 (1986).

${ }^{2} \mathrm{H}$. Nalwa, Handbook of Low and High Dielectric Constant Materials and Their Applications (Academic Press, London, 1999).

${ }^{3}$ Y. Rao, S. Ogitani, P. Kohl and C. P. Wong, Novel polymerceramic nanocomposite based on high dielectric constant epoxy formula for embedded capacitor application, J. Appl. Polym. Sci. 83, 1084 (2002).

${ }^{4}$ Z. Y. Cheng and Q. M. Zhang. Field actuated electroactive polymers, Mater. Res. Bull. 33, 183 (2008).

${ }^{5}$ J. Lu and C. P. Wong, Recent advances in high- $k$ nanocomposite materials for embedded capacitor applications, IEEE Trans. Dielect. Electr. Insul. 15, 1322 (2008).

${ }^{6}$ Y. Rao and C. P. Wong, Material characterization of a high-dielectric-constant polymer-ceramic composite for embedded capacitor for RF applications, J. Appl. Polym. Sci. 92, 2228 (2004). ${ }^{7}$ C. B. Carter and M. G. Norton, Ceramic Materials: Science and Engineering (Spring, New York, 2007).

${ }^{8}$ H. S. Nalwa, Ferroelectric Polymers: Chemistry, Physics, and Applications (Marcel Dekker Inc., New York, 1995).

${ }^{9}$ Z. M. Dang J. K. Yuan, J. W. Zha, T. Zhou, S. T. Li and G. H. Hu, Fundamentals, processes and applications of high-permittivity polymer-matrix composites, Prog. Mater. Sci. 57, 660 (2012).

${ }^{10}$ L. Zhang and Z.-Y. Cheng, Development of polymer-based 0-3 composites with high dielectric constant, J. Adv. Dielect. 1, 389 (2011).

${ }^{11}$ C. W. Nan, Y. Shen and J. Ma, Physical properties of composites near percolation, Annu. Rev. Mater. Res. 40, 131 (2010).

${ }^{12}$ Z. M. Dang, H. Y. Wang, B. Peng and C. W. Nan, Effect of $\mathrm{BaTiO}_{3}$ size on dielectric property of $\mathrm{BaTiO}_{3} / \mathrm{PVDF}$ composites, J. Electroceram. 21, 381 (2008).

${ }^{13}$ Z. M. Dang, Y. Zheng and H. P. Xu, Effect of ceramic particle sizes on morphology and dielectric properties in the $\mathrm{BaTiO}_{3} /$ polystyrene composites, J. Appl. Polym. Sci. 110, 3473 (2008).

${ }^{14}$ D. H. Kuo, C. C. Chang, T. Y. Sub, W. K. Wang and B. Y. Lin, Dielectric properties of three ceramic/epoxy composites, Mater. Chem. Phys. 85, 201 (2004).

${ }^{15}$ Y. Bai, Z.-Y. Cheng, V. Bharti, H. S. Xu and Q. M. Zhang, Highdielectric-constant ceramic-powder polymer composites, Appl. Phys. Lett. 76, 3804 (2000).

${ }^{16}$ L. J. Dong, C. X. Xiong, H. Y. Quan and G. Z. Zhao, Polyvinylbutyral/lead zirconate titanates composites with high dielectric constant and low dielectric loss, Scr. Mater. 55, 835 (2006).

${ }^{17}$ Z.-Y. Cheng, R. S. Katiyar, X. Yao and A. S. Bhalla, Temperature dependence of the dielectric constant of relaxor ferroelectrics, Phys. Rev. B 57, 8166 (1998). 
${ }^{18}$ M. A. Subramanian, D. Li, N. Duan, B. A. Reisner and A. W. Sleight, High dielectric constant in $\mathrm{ACu}_{3} \mathrm{Ti}_{4} \mathrm{O}_{12}$ and $\mathrm{ACu}_{3} \mathrm{Ti}_{3-}$ $\mathrm{FeO}_{12}$ phases, J. Solid State Chem. 151, 323 (2000).

${ }^{19}$ M. A. Subramaniana and A. W. Sleight, $\mathrm{ACu}_{3} \mathrm{Ti}_{4} \mathrm{O}_{12}$ and $\mathrm{ACu}_{3} \mathrm{Ru}_{4} \mathrm{O}_{12}$ perovskites: High dielectric constants and valence degeneracy, Solid State Sci. 4, 347 (2002).

${ }^{20}$ M. D. Arbatti, X. B. Shan and Z.-Y. Cheng, Ceramic-polymer composites with high dielectric constant, Adv. Mater. 19, 1369 (2007).

${ }^{21}$ L. Zhang, X. B. Shan, P. X. Wu and Z.-Y. Cheng, Dielectric characteristics of $\mathrm{CaCu}_{3} \mathrm{Ti}_{4} \mathrm{O}_{12} / \mathrm{P}$ (VDF-TrFE) nanocomposites, J. Appl. Phys. A 107, 597 (2012)

${ }^{22}$ L. Zhang, P. X. Wu, Y. T. Li and Z.-Y. Cheng, Preparation process and dielectric properties of $\mathrm{Ba}_{0.5} \mathrm{Sr}_{0.5} \mathrm{TiO}_{3}-\mathrm{P}(\mathrm{VDF}-\mathrm{CTFE})$ nanocomposites, Compos. Part B-Eng. 56, 284 (2014).

${ }^{23}$ Z. M. Dang, T. Zhou, S. H. Yao, J. K. Yuan, J. W. Zha, H. T. Song, J. Y. Li, Q. Chen, W. T. Yang and J. B. Bai, Advanced calcium copper titanate/polyimide functional hybrid films with high dielectric permittivity, Adv. Mater. 21, 2077 (2009).

${ }^{24}$ X. B. Shan, L. Zhang, X. Q. Yang and Z.-Y. Cheng, Dielectric composites with a high and temperature-independent dielectric constant, J. Adv. Ceram. 1(4), 310 (2012).

${ }^{25} \mathrm{M}$. T. Clarkson, Electrical conductivity and permittivity measurements near the percolation transition in a microemulsion. II. Interpretation, Phys. Rev. A 37, 2079 (1988).

${ }^{26}$ J. K. Yuan, Z. M. Dang and J. B. Bai, Unique dielectric properties in polyaniline/poly(vinylidene fluoride) composites induced by temperature variation, Phys. Stat. Sol. 2, 233 (2008).

${ }^{27}$ J. Macutkevic, D. Seliuta, G. Valušis, J. Banys, V. Kuznetsov, S. Moseenkov and O. Shenderova, High dielectric permittivity of percolative composites based on onion-like carbon, Appl. Phys. Lett. 95, 112901 (2009).
${ }^{28}$ L. Zhang, W. Wang, X. G. Wang, P. Bass and Z.-Y. Cheng, Metalpolymer nanocomposites with high percolation threshold and high dielectric constant, Appl. Phys. Lett. 103, 232903 (2013).

${ }^{29}$ L. Zhang, P. Bass and Z.-Y. Cheng, Revisiting the percolation phenomena in dielectric composites with conducting fillers, Appl. Phys. Lett. 105, 042905 (2014).

${ }^{30}$ Z. M. Dang, B. Xia, S. H. Yao, M. J. Jiang, H. T. Song, L. Q. Zhang and D. Xie, High-dielectric-permittivity high-elasticity three-component nanocomposites with low percolation threshold and low dielectric loss, Appl. Phys. Lett. 94, 042902 (2009).

${ }^{31}$ Z. M. Dang, B. Peng, D. Xie, S. H. Yao, M. J. Jiang and J. B. Bai, High dielectric permittivity silver/polyimide composite films with excellent thermal stability, Appl. Phys. Lett. 92, 112910 (2008).

${ }^{32}$ Z. M. Dang J. P. Wu, H. P. Xu, S. H. Yao, M. J. Jiang and J. B. Bai, Dielectric properties of upright carbon fiber filled poly(vinylidene fluoride) composite with low percolation threshold and weak temperature dependence, Appl. Phys. Lett. 91, 072912 (2007).

${ }^{33}$ Z. M. Dang, L. Wang, Y. Yin, Q. Zhang and Q. Q. Lei, Giant dielectric permittivities in functionalized carbon-nanotube/electroactive-polymer nanocomposites, Adv. Mater. 19, 852 (2007).

${ }^{34}$ L. Wang and Z. M. Dang, Carbon nanotube composites with high dielectric constant at low percolation threshold, Appl. Phys. Lett. 87, 042903 (2005).

${ }^{35}$ D. J. Bergman and Y. Imry, Critical behavior of the complex dielectric constant near the percolation threshold of a heterogeneous material, Phys. Rev. Lett. 39, 1222 (1977).

${ }^{36}$ I. Webman, J. Jortner and M. H. Cohen, Critical exponents for percolation conductivity in resistor networks, Phys. Rev. B 16, 2593 (1977) 